\title{
Conductance quantization in graphene nanoribbons: adiabatic approximation
}

\author{
M.I. Katsnelson ${ }^{\mathrm{a}}$ \\ Institute for Molecules and Materials, Radboud University Nijmegen, 6525 ED Nijmegen, The Netherlands \\ Received 5 March 2007 / Received in final form 14 May 2007 \\ Published online 15 June 2007 - (c) EDP Sciences, Società Italiana di Fisica, Springer-Verlag 2007
}

\begin{abstract}
A theory of electron states for graphene nanoribbons with a smoothly varying width is developed. It is demonstrated that the standard adiabatic approximation allowing to neglect the mixing of different standing waves is more restrictive for the massless Dirac fermions in graphene than for the conventional electron gas. For the case of zigzag boundary conditions, one can expect a well-pronounced conductance quantization only for highly excited states. This difference is related to the relativistic Zitterbewegung effect in graphene.
\end{abstract}

PACS. 73.43.Cd Theory and modeling - 81.05.Uw Carbon, diamond, graphite - 03.65.Pm Relativistic wave equations

The experimental discovery of a truly two-dimensional allotrope of carbon, graphene $[1,2]$ and of the massless Dirac character of its electronic energy spectrum $[3,4]$ has initiated an enormously growing interest in this field (for review, see Refs. $[5,6]$ ). One of the most exciting aspects of the problem is the hope to develop novel carbon-based electronics. Very recently, the experimental realization of quantum dots [5] and carbon nanoribbons $[7,8]$ has been announced, the former demonstrating single-electron transistor (SET) effect [5].

The conductance quantization in the ballistic regime $[9-13]$ is one of the most important physical phenomena determining the functioning of such nanodevices. It was considered recently for the case of ideal graphene stripe [14] and for the case of confinement due to a smooth external electrostatic potential [15]. The experimental situation $[5,7,8]$ corresponds rather to the case of electron confinement due to a curvilinear shape of the graphene samples than to an external field. The description of the penetration of electron waves through constrictions in the nanoribbons requires a different theoretical approach. Numerical calculations of electronic transport in graphene nanoribbons demonstrating a very interesting "valley filter" effect have been recently published [16]. However, a general theoretical analysis of the situation is still absent. Here we present an analytical theory of conductance quantization in graphene nanoribbons based on the adiabatic approximation $[10$, 12]. The latter means a separation of the electron motion in the directions perpendicular and along the stripe. For nonrelativistic electrons the adiabatic approxima-

\footnotetext{
a e-mail: M.Katsnelson@science.ru.nl
}

tion requires only the smoothness of the shape of the stripe boundary and results in the quantization of the conductance. For graphene, the situation turns out to be essentially dependent on the boundary conditions. It will be shown that for the zigzag boundaries this theory is essentially different from that for nonrelativistic electrons and, in general, there is no reason to expect an adiabatic regime and well-pronounced conductance jumps for the lowest states of the ribbon.

The two-component wave function $(u, v)$ for charge carriers in graphene with wave vectors close to the $K$ point is described by the Dirac equation

$$
\begin{aligned}
& \left(\frac{\partial}{\partial x}+i \frac{\partial}{\partial y}\right) u=i k v \\
& \left(\frac{\partial}{\partial x}-i \frac{\partial}{\partial y}\right) v=i k u
\end{aligned}
$$

where $k=E / \hbar v_{F}, E$ is the electron energy and $v_{F} \simeq$ $10^{6} \mathrm{~m} / \mathrm{s}$ is the Fermi velocity [3,4]; for the other valley $K^{\prime}$ the signs before $\partial / \partial y$ are opposite. Let us consider first the case of a uniform graphene strip of width $L$ along the $y$-axis, $|y|<L / 2$. To specify the problem one has to choose boundary conditions at the edges [17].

We start with the case of zigzag edges where $u(y=-L / 2)=0, v(y=L / 2)=0$. The energy spectrum is discrete, $E_{j}=\hbar v_{F} k_{j}$ where

$$
k_{j}=\frac{\pi j}{L}, j= \pm \frac{1}{2}, \pm \frac{3}{2}, \ldots
$$


and the wave functions have the form

$$
\begin{aligned}
& u_{j}(y)=\frac{1}{\sqrt{L}} \cos k_{j}(y-L / 2), \\
& v_{j}(y)=-\frac{1}{\sqrt{L}} \sin k_{j}(y-L / 2) .
\end{aligned}
$$

Consider now the case of a smoothly varying strip width, $L \rightarrow L(x),|d L / d x| \ll 1$. Following a general scheme $[10,12]$ one can try a solution of equation (1) as an expansion

$$
\begin{aligned}
& u(x, y)=\sum_{j} c_{j}(x) u_{j}^{(x)}(y) \\
& v(x, y)=\sum_{j} c_{j}(x) v_{j}^{(x)}(y)
\end{aligned}
$$

where $u^{(x)}, v^{(x)}$ are the functions (3) with the replacement $L \rightarrow L(x)$. The functions (4) satisfy by construction the boundary conditions. By substituting the expansion (4) into equation (1), multiplying the first equation by $\left\langle v_{j}\right|$ and the second one by $\left\langle u_{j}\right|$ one finds:

$$
\begin{aligned}
& \sum_{j^{\prime}}\left[\frac{d c_{j^{\prime}}}{d x}\left\langle v_{j} \mid v_{j^{\prime}}\right\rangle+c_{j^{\prime}}\left\langle v_{j} \mid \frac{d v_{j^{\prime}}}{d x}\right\rangle\right]= i \sum_{j^{\prime}}\left(k-k_{j^{\prime}}\right) \\
& \sum_{j^{\prime}}\left[\frac{d c_{j^{\prime}}}{d x}\left\langle v_{j} \mid u_{j^{\prime}}\right\rangle\right. \\
&\left.\left.u_{j^{\prime}}\right\rangle+c_{j^{\prime}}\left\langle u_{j} \mid \frac{d u_{j^{\prime}}}{d x}\right\rangle\right]= i \sum_{j^{\prime}}\left(k-k_{j^{\prime}}\right) \\
& c_{j^{\prime}}\left\langle u_{j} \mid v_{j^{\prime}}\right\rangle .
\end{aligned}
$$

This equation is formally exact. As a first step to the adiabatic approximation, one should neglect the terms with $\left\langle v_{j} \mid \frac{d v_{j^{\prime}}}{d x}\right\rangle$ and $\left\langle u_{j} \mid \frac{d u_{j^{\prime}}}{d x}\right\rangle$ which is justified by the smallness of $d L / d x$, as in the case of nonrelativistic electrons [10,12].

To proceed further we need to calculate the overlap integrals $\left\langle\phi_{1} \mid \phi_{2}\right\rangle=\int_{-L / 2}^{L / 2} d y \phi_{1}^{*} \phi_{2}$ for different basis functions:

$$
\begin{aligned}
& \left\langle u_{j} \mid u_{j^{\prime}}\right\rangle=\frac{1}{2}\left(\delta_{j j^{\prime}}+\delta_{j,-j^{\prime}}\right), \\
& \left\langle v_{j} \mid v_{j^{\prime}}\right\rangle=\frac{1}{2}\left(\delta_{j j^{\prime}}-\delta_{j,-j^{\prime}}\right), \\
& \left\langle u_{j} \mid v_{j^{\prime}}\right\rangle=\left\langle v_{j^{\prime}} \mid u_{j}\right\rangle= \begin{cases}-\frac{1}{\pi\left(j^{\prime}-j\right)}, & j^{\prime}-j=2 n+1, \\
-\frac{1}{\pi\left(j^{\prime}+j\right)}, & j^{\prime}-j=2 n,\end{cases}
\end{aligned}
$$

where $n$ is integer. Substituting equation (6) into equation (5) and neglecting the nonadiabatic terms with the matrix elements of the operator $d / d x$, we obtain after simple transformations:

$$
\frac{d c_{j}(x)}{d x}=-\frac{2 i}{\pi} \sum_{j^{\prime}}^{\prime} \frac{\left[k-k_{j^{\prime}}(x)\right]}{j+j^{\prime}} c_{j^{\prime}}(x)
$$

where the sum is over all $j^{\prime}$ such that $j^{\prime}-j$ is even.
Until now we did transformations and approximations which are identical to those used in the case of nonrelativistic electrons. However, we still have a coupling between different standing waves so we still cannot prove that the electron transmission through the constriction is adiabatic. To prove the latter we need one more step, namely, a transition from the discrete variable $j$ to real one and a replacement of the sums by integrals in the right-hand-side of equation $(7): \sum_{j}^{\prime} \ldots \rightarrow \frac{1}{2} \mathcal{P} \int d j \ldots$ where $\mathcal{P}$ is the symbol of principle value. This step is justified by assuming that $k L \gg 1$, i.e., only for highly excited states. For the low-lying electron standing waves it is difficult to see any way to simplify essentially the set of equations (7) for the coupled states.

For any function $f(z)$ analytical in the upper (lower) complex half-plane one has

$$
\int_{-\infty}^{\infty} d x f(x) \frac{1}{x-x_{1} \pm i 0}=0
$$

or, equivalently,

$$
\int_{-\infty}^{\infty} d x f(x) \frac{\mathcal{P}}{x-x_{1}}= \pm i \pi f\left(x_{1}\right)
$$

Assuming that $c_{j}(x)$ is analytical in the lower halfplane as a function of complex variable $j$ one obtains, instead of equation (7)

$$
\frac{d c_{j}(x)}{d x}=\left[k+k_{j}(x)\right] c_{-j}(x) .
$$

Similar, taking into account that $c_{-j}(x)$ is analytical in the upper halfplane as a function of complex variable $j$ we have

$$
\frac{d c_{-j}(x)}{d x}=\left[k_{j}(x)-k\right] c_{j}(x) .
$$

At last, differentiating equation (10) with respect to $x$, neglecting the derivatives of $k_{j}(x)$ due to the smallness of $d L / d x$ and taking into account equation (11) we find

$$
\frac{d^{2} c_{j}(x)}{d x^{2}}+\left[k^{2}-k_{j}^{2}(x)\right] c_{j}(x)=0
$$

Further analysis completely follows that for the nonrelativistic case [10] where $k^{2}$ and $k_{j}^{2}(x)$ play the roles of energy and potential, respectively. The potential is quasiclassical for the case of smoothly varying $L(x)$. Therefore, the transmission coefficient is very close to one if the electron energy exceeds the energy of the $j$ th level in the narrowest place of the constriction, and exponentially small, otherwise. Standard arguments based on the Landauer formula [9-13] prove the conductance quantization in this situation.

At the same time, for the lowest energy levels the replacement of sums by integrals in equation (10) cannot be justified and thus the states with different $j$ 's are in general coupled even for a smooth constriction $(|d L / d x| \ll 1)$. 
Therefore electron motion along the stripe is strongly coupled with that in the perpendicular direction and different electron standing waves are essentially entangled. In this situation there is no general reason to expect sharp jumps and well-defined plateaus in the energy dependence of the conductance. This means that the criterion of adiabatic approximation is more restrictive for the case of Dirac electrons than for the nonrelativistic ones. The formal reason is an overlap between components of the wave functions with different pseudospins or, equivalently, between hole component of the state $j$ with the electron component of the state $j^{\prime} \neq j$. This coupling is a reminiscence of the Zitterbewegung of Dirac electrons determining the finite conductivity and anomalous shot noise in graphene in the limit of small charge carrier concentration $[18,19]$. Effectively, it works as a kind of intrinsic "disorder" and therefore it is not surprising that it destroys the ballistic regime near the Dirac point. Interestingly, the kinetic equation that takes into account the Zitterbewegung effects also contains some "P -terms" which are absent in the standard Boltzmann equation; these terms become irrelevant for large enough Fermi energy [20].

Consider now the case of armchair edges. The boundary conditions are coupled the components of Dirac spinors at $K$ valley $u, v$ with those at $K^{\prime}$ valley $\bar{u}, \bar{v}$ :

$$
\begin{aligned}
u(-L / 2) & =\bar{u}(-L / 2), \\
v(-L / 2) & =\bar{v}(-L / 2), \\
u(L / 2) & =e^{2 \pi i \nu} \bar{u}(L / 2), \\
v(L / 2) & =e^{2 \pi i \nu} \bar{v}(L / 2),
\end{aligned}
$$

where $\nu=0, \pm 2 / 3$, depending on the number of rows in the strip [17]. The eigenmodes in that case are just plane waves $[17]$

$$
\begin{aligned}
u_{j}(y) & =-i v_{j}(y)=\frac{1}{\sqrt{2 L}} \exp \left(i k_{j} y\right), \\
\bar{u}_{j}(y) & =-i \bar{v}_{j}(y)=\frac{1}{\sqrt{2 L}} \exp \left(-i k_{j} y\right), \\
k_{j} & =(j+\nu) \pi / L, j=0, \pm 1, \pm 2, \ldots
\end{aligned}
$$

A general solution satisfying the boundary conditions (13) can be probed as

$$
\begin{aligned}
& u(x, y)=\sum_{j} c_{j}(x) \exp \left[i k_{j}(x) y\right], \\
& v(x, y)=i \sum_{j} b_{j}(x) \exp \left[i k_{j}(x) y\right], \\
& \bar{u}(x, y)=\sum_{j} c_{j}(x) \exp \left[i k_{j}(x)(L(x)-y)\right], \\
& \bar{v}(x, y)=i \sum_{j} b_{j}(x) \exp \left[i k_{j}(x)(L(x)-y)\right] .
\end{aligned}
$$

Substituting this into the Dirac equation (1) one obtains

$$
\begin{aligned}
& \sum_{j} \exp \left[i k_{j}(x) y\right]\left\{\frac{d c_{j}}{d x}+\left(k b_{j}-k_{j} c_{j}\right)+i \frac{d k_{j}}{d x} y c_{j}\right\}=0 \\
& \sum_{j} \exp \left[i k_{j}(x) y\right]\left\{\frac{d b_{j}}{d x}+\left(k_{j} b_{j}-k c_{j}\right)+i \frac{d k_{j}}{d x} y b_{j}\right\}=0 .
\end{aligned}
$$

Let us neglect first the nonadiabatic terms proportional to $d k_{j} / d x$ in these equations. They will be satisfied for sure if all coefficients at the plane waves vanish, which is equivalent to the set of equations

$$
\begin{aligned}
& \frac{d\left(c_{j}+b_{j}\right)}{d x}+\left(k+k_{j}\right)\left(b_{j}-c_{j}\right)=0, \\
& \frac{d\left(b_{j}-c_{j}\right)}{d x}+\left(k_{j}-k\right)\left(c_{j}+b_{j}\right)=0 .
\end{aligned}
$$

Differentiating them with respect to $x$ and neglecting, again, the derivatives of $k_{j}$ we find the effective Schrödinger equation (12) and the same equation for $b_{j}$. Thus, in contrast with the case of zigzag edges, for the armchair edges a standard picture of conductance quantization should be valid for all states, similar to nonrelativistic electron gas.

However, there is another problem which makes the adiabatic approximation for this case problematic. The wave numbers (15) can depend on $x$ not only due to the stripe length but also due to different number of rows in the stripe which makes $d \nu / d x$ a source of sharp random potential. It is very difficult to investigate this effect analytically in the framework of the approach under consideration. It was argued recently based on numerical results and qualitative considerations that this kind of randomness should be of crucial importance for the graphene nanoribbons with the armchair edges [21].

It would be very interesting to check experimentally the possible difference in the conductance behavior for the nanoribbons with zigzag and armchair edges. For the former case, the theory predicts essential difference of behavior at the crossing of low-lying and highly excited energy levels in the quantum point contact situation, that is, for a narrow constriction of the graphene nanoribbons.

I am thankful to Andre Geim, Kostya Novoselov, Carlo Beenakker, and Annalisa Fasolino for helpful discussions. This work was supported by the Stichting voor Fundamenteel Onderzoek der Materie (FOM), The Netherlands.

\section{References}

1. K.S. Novoselov, D. Jiang, F. Schedin, T.J. Booth, V.V. Khotkevich, S.M. Morozov, A.K. Geim, PNAS 102, 10451 (2005)

2. K.S. Novoselov, A.K. Geim, S.V. Morozov, D. Jiang, Y. Zhang, S.V. Dubonos, I.V. Grigorieva, A.A. Firsov, Science 306, 666 (2004) 
3. K.S. Novoselov, A.K. Geim, S.V. Morozov, D. Jiang, M.I. Katsnelson, I.V. Grigorieva, S.V. Dubonos, A.A. Firsov, Nature 438, 197 (2005)

4. Y. Zhang, Y.-W. Tan, H.L. Stormer, P. Kim, Nature 438, $201(2005)$

5. A.K. Geim, K.S. Novoselov, Nature Mater. 6, 183 (2007)

6. M.I. Katsnelson, Mater. Today 10, Issue 1\&2, 20 (2007)

7. Z. Chen, Y.-M. Lin, M. J. Rooks, P. Avouris, e-print arXiv: cond-mat/0701599

8. M.Y. Han, B. Özyilmaz, Y. Zhang, P. Kim, e-print arXiv cond-mat/0702511

9. C.W.J. Beenakker, H. van Houten, Solid State Phys. 44, 1 (1991)

10. L.I. Glazman, G.B. Lesovik, D.E. Khmelnitskii, R.I. Shekhter, Pis'ma ZhETF 48, 218 (1988)
11. M.C. Payne, J. Phys.: Condens. Matter 1, 4939 (1989)

12. A. Yakoby, Y. Imry, Phys. Rev. B 41, 5341 (1990)

13. M. Yoselin, M. Kaveh, Phys. Rev. Lett. 64, 2819 (1990)

14. N.M.R. Peres, A.H. Castro Neto, F. Guinea, Phys. Rev. B 73, 195411 (2006)

15. P.G. Silvestrov, K.B. Efetov, Phys. Rev. Lett. 98, 016802 (2007)

16. A. Rycerz, J. Tworzydlo, C.W.J. Beenakker, Nature Phys. 3, 172 (2007)

17. L. Brey, H.A. Fertig, Phys. Rev. B 73, 235411 (2006)

18. M.I. Katsnelson, Eur. Phys. J. B 51, 157 (2006)

19. J. Tworzydlo, B. Trauzettel, M. Titov, A. Rycerz, C.W.J. Beenakker, Phys. Rev. Lett. 96, 246802 (2006)

20. M.I. Auslender, M.I. Katsnelson (to be published)

21. I. Martin, Ya.M. Blanter, e-print arXiv:0705.0532 